\title{
Capecitabine Plus Bevacizumab for Cardiac Metastasis of Sigmoid Colon Cancer: Case Report and Literature Review
}

\author{
SAWAKO HIROI ${ }^{1}$, MASASHI MIGUCHI ${ }^{1}$, SATOSHI IKEDA ${ }^{1}$, HIDEKI NAKAHARA ${ }^{1}$, \\ KATSUNORI SHINOZAKI ${ }^{2}$, TAKASHI NISHISAKA ${ }^{3}$, HIROYUKI EGI ${ }^{4}$ and TOSHIYUKI ITAMOTO ${ }^{1,4}$ \\ ${ }^{1}$ Department of Gastroenterological Surgery, Hiroshima Prefectural Hospital, Hiroshima, Japan; \\ ${ }^{2}$ Division of Clinical Oncology, Hiroshima Prefectural Hospital, Hiroshima, Japan; \\ ${ }^{3}$ Department of Pathology Clinical Laboratory, Hiroshima Prefectural Hospital, Hiroshima, Japan; \\ ${ }^{4}$ Department of Gastroenterological and Transplant Surgery, Applied Life Sciences, \\ Institute of Biomedical and Health Sciences, Hiroshima University, Hiroshima, Japan
}

\begin{abstract}
Background/Aim: Right ventricular cardiac metastasis from colorectal cancer (CRC) is rare and clinically silent. There is no standardised treatment. To date, only twelve cases have been reported in the literature. This is a case report and literature review of right ventricular cardiac metastasis from CRC. Case Report: A 75-year-old woman with a history of CRC treated with sigmoidectomy followed by liver and lung metastasectomy presented with a right ventricle tumour. Biopsy showed metastatic adenocarcinoma not suitable for resection because multiple lung metastases coexisted. The metastases were controlled for a prolonged duration by chemotherapy with capecitabine plus bevacizumab. According to the review of 13 cases, the median age of metastatic CRC that involves the right ventricle is 71 years and the primary site is half the colon and rectum. Half of cases have non-cardiac metastases at cardiac metastasis diagnosis. Chemotherapy is more suitable than resection in cases with metastases other than heart because resection of the right ventricle has a high risk. Conclusion: Cardiac right ventricular metastasis from CRC can be controlled by capecitabine plus bevacizumab.
\end{abstract}

Metastases from colorectal cancer (CRC) can occur either by lymphatic or hematogenous spreading, and the sites most commonly involved are the lymph nodes, liver, and lung.

This article is freely accessible online.

Correspondence to: Masashi Miguchi, Department of Gastroenterological Surgery, Hiroshima Prefectural Hospital, 1-5-54 Ujina-kanda, Minami-ku, Hiroshima, 734-8530, Japan. Tel: +81 822541818, Fax: +81 822538274, e-mail: miguchima0815@gmail.com

Key Words: Bevacizumab, capecitabine, cardiac metastasis, colorectal cancer, right ventricle.
Cardiac metastasis from CRC is an extremely rare event and the incidence is probably underestimated. A large autopsy series reported that colon carcinoma metastases to the heart account for $1.2 \%$, with a prevalence of $2 \%$, of all metastatic neoplasms (1). The reasons for the limited number of reported cases are that cardiac tumours are usually asymptomatic and do not present themselves. A progressive metastatic mass of the heart occasionally causes acute heart failure or superior vena cava syndrome, resulting in sudden death $(2,3)$. Such masses are often associated with non-specific symptoms, including those of right-sided heart failure, dyspnoea, arrhythmias, pulmonary thromboembolism, and tumour thrombus (4). With regard to the frequency of cardiac metastases from any malignancy, the epicardium (75.5\%), followed by the myocardium (38.2\%) and endocardium $(15.5 \%)$ has been reported to be involved (5). In this report, cardiac metastasis refers to the involvement of parts other than the epicardium. Herein, we present a case of a patient with right ventricular cardiac metastasis from adenocarcinoma of the sigmoid colon who was effectively treated with chemotherapy and review reports to date regarding CRC metastases to the right ventricle of the heart, their diagnosis, treatments, and prognosis.

\section{Case Report}

In July 2013, a 70-year-old woman underwent sigmoidectomy for moderately differentiated adeno-carcinoma with wild-type $R A S / B R A F$. The presurgical radiological staging was deemed negative for distant metastases. The pathological stage was pT3N0M0 (stage IIA). The patient did not receive adjuvant therapy and entered the following scheduled clinical follow-up program: regular physical examinations and carcinoembryonic antigen test every 3 months and whole-body computed tomography (CT) every 6 months. A follow-up CT in February 2015 showed metachronous resectable liver metastasis. She 

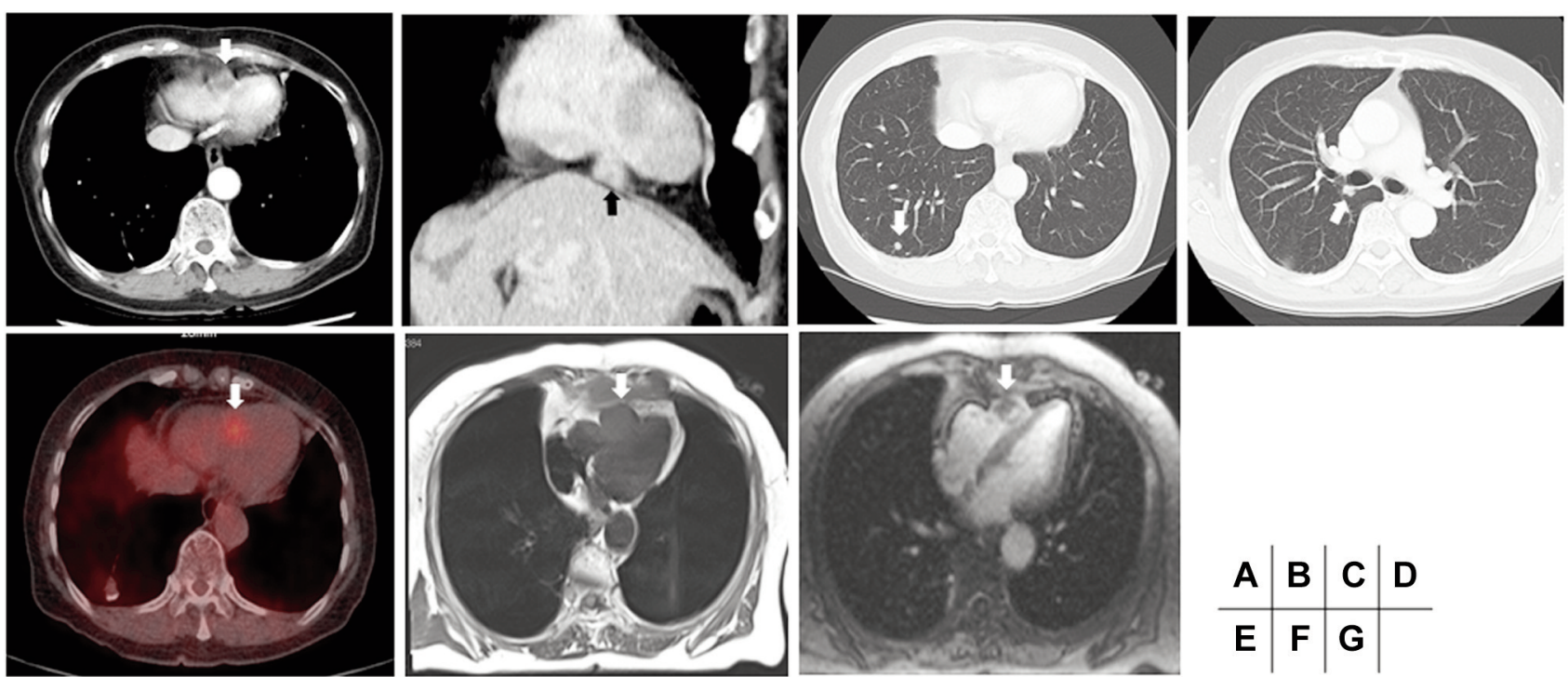

Figure 1. (A, B) Computed tomography $(C T)$ showing a tumour, measuring approximately $20 \mathrm{~mm}$, in the right ventricle of the heart. $(C, D) C T$ showing two small lesions, suspected to be metastasis from the colorectal cancer in the left lung. (E) ${ }^{18}$ F-Fluorodeoxyglucose positron emission tomography showing a significant fluorodeoxyglucose uptake in the right ventricular mass (SUV $\left.\max _{1}: 3.2\right)$. (F) T1-weighted magnetic resonance imaging (MRI) showing a slightly high intense mass, measuring $20 \mathrm{~mm}$, compared to the intact myocardium. (G) T2-weighted MRI showing the right ventricle with an irregular, isointense mass.

was treated with seven courses of neoadjuvant chemotherapy with CapeOX $\left(1,000 \mathrm{mg} / \mathrm{m}^{2}\right.$ capecitabine and $130 \mathrm{mg} / \mathrm{m}^{2}$ oxaliplatin) plus bevacizumab $(7.5 \mathrm{mg} / \mathrm{kg})$. Segmental liver resection was performed in December 2015. Because CT in June 2016 showed metachronous resectable lung metastasis, partial resection of the right lung was performed in August 2016. After the operation, she was treated with four courses of adjuvant chemotherapy with CapeOX. In February 2018, carcinoembryonic antigen levels were found to be elevated at $8.5 \mathrm{ng} / \mathrm{ml}$. In June 2018, a CT showed a cardiac tumour approximately $20 \mathrm{~mm}$ in size (Figure 1A and B) and multiple unilateral pulmonary tumours (Figure $1 \mathrm{C}$ and D). Furthermore, ${ }^{18}$ F-fluorodeoxyglucose positron emission tomography revealed significant accumulation in both cardiac and pulmonary tumours (Figure $1 \mathrm{E}$ ). With regard to the cardiac tumour, magnetic resonance imaging showed an irregular 20$\mathrm{mm}$ mass that was isointense on T1-weighted images, slightly more intense than the intact myocardium on T2-weighted images (Figure $1 \mathrm{~F}$ and $\mathrm{G}$ ), and characterised by a delayed enhancement. The patient underwent cardiac catheterisation with biopsy of the right atrial mass, which did not show any evidence of tumour cells. The cardiac catheterisation also revealed no intracardiac tumour or cardiac dysfunction. In July 2018, a limited excision biopsy was performed by minimally invasive cardiac surgery. A hard tumour of approximately $3 \mathrm{~cm}$ was detected at the apical part of the right ventricle during surgery and incisional biopsy of the tumour was performed. Microscopic pathological examination of the biopsy tissue confirmed metastatic adenocarcinoma, the histological features of which were similar to those of primary adenocarcinoma of the sigmoid colon. The tumour was deemed not suitable for resection and systemic chemotherapy was considered because multiple lung metastases coexisted. As the patient was 75 years old with an Eastern Cooperative Oncology Group performance status of 1 , capecitabine and bevacizumab were administered. There was no grade 3-4 adverse event; therefore, the chemotherapy regimen was continued as scheduled with no dose reductions or discontinuation until June 2020 to ensure long-lasting stable disease (Figure 2). The clinical course since primary sigmoidectomy is shown in Figure 3. Written informed consent for patient information and images to be published was provided by the patient.

\section{Discussion}

We present a case of cardiac metastasis from CRC 5 years following primary tumour and hepatectomy/pulmonary resection for liver/pulmonary metastasis. The patient's disease could be controlled for a prolonged duration by chemotherapy with capecitabine plus bevacizumab.

There is no standardized approach or treatment for managing patients with cardiac metastases from CRC and the prognosis for patients with the metastatic cardiac tumours is unknown. Concerning the treatment of cardiac metastases from any malignancy, several reports emphasise the role of surgery based on significant improvements in survival times. 

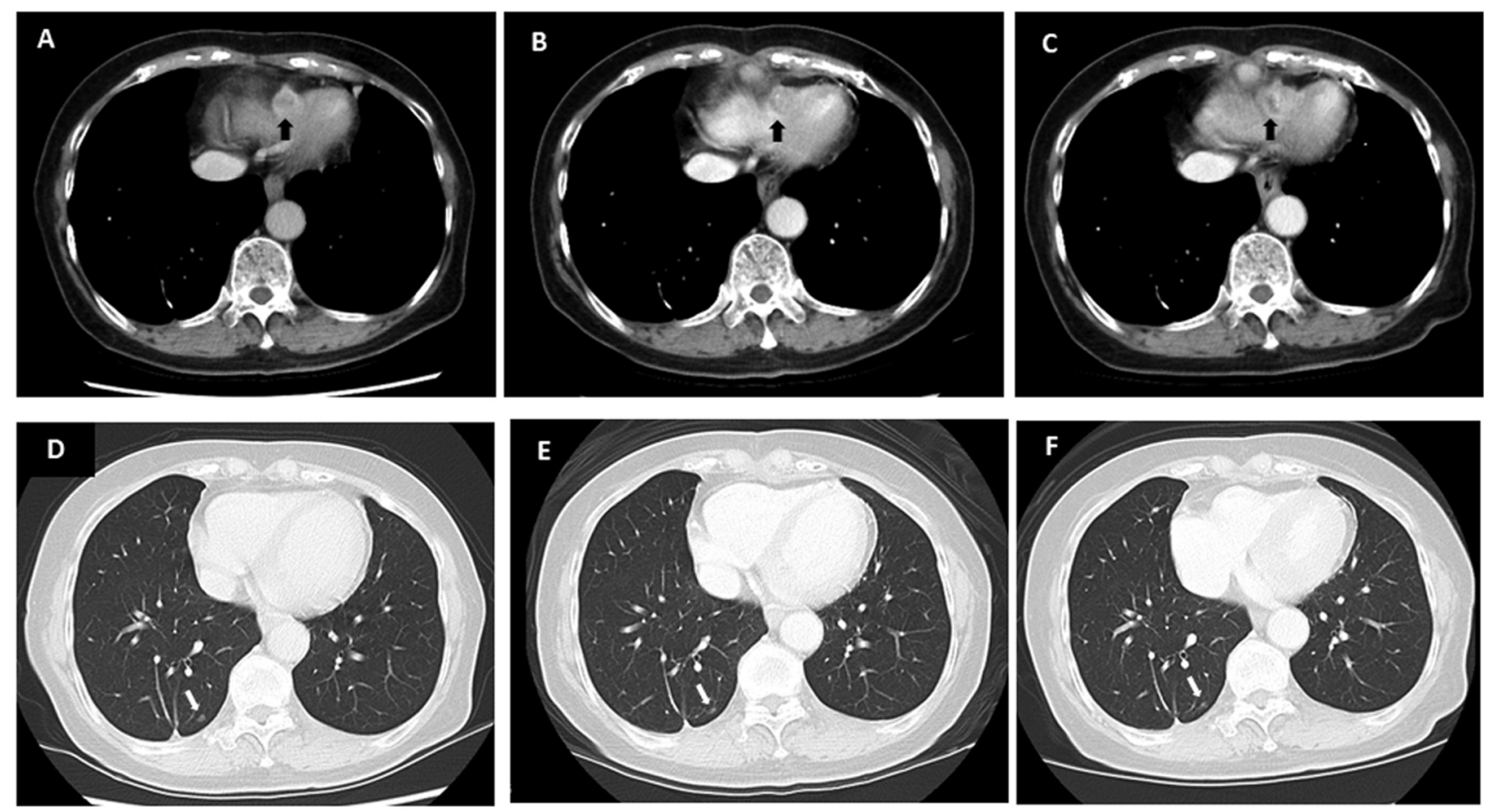

Figure 2. Changes in metastatic lesions evaluated by computed tomography $(C T)$ during capecitabine plus bevacizumab chemotherapy. The metastatic lesions remained approximately the same size. (A-C) CT findings of cardiac ventricular metastasis, $(D-F) C T$ findings of one of the left lung metastases. $(A, D)$ Baseline CT findings at the start of chemotherapy. $(B, E) C T$ findings at 9 months after starting chemotherapy. (C, $F) C T$ findings at 17 months after starting chemotherapy.

Murphy et al. performed operations on 19 patients with cardiac metastases and reported an operative survival of $68.4 \%$, with a significant improvement in the quality of life and prolonged life expectancy (the average survival was 3.2 years for five patients) (6), which has confirmed by other reports $(7,8)$. Surgery may be indicated for palliation, when symptoms of haemodynamic compromise secondary to intracavitary tumour growth outweigh the risk of operative death, allowing more time for treatment with chemotherapy or radiation and increasing life expectancy (7). However, the potential survival benefit from cardiac surgery may be counterbalanced by perioperative morbidity and mortality. Therefore, indications for surgery in patients with metastatic cardiac tumours must be carefully considered, particularly for asymptomatic elderly patients or those with other metastatic lesions. Currently, little evidence is available regarding the efficacy of chemotherapy against cardiac metastasis from CRC; however, recently, the efficacy of combination therapy using cytotoxic drugs and molecular targeted agents such as anti-EGFR antibodies against cardiac metastasis from wild-type RAS CRC was reported (9). In the case of our patient, surgical resection of the cardiac metastasis was considered dangerous and unwarranted because of the presence of progressive lung metastasis.
Furthermore, because the patient was aged $\geq 75$ years, had a poor Eastern Cooperative Oncology Group performance status, and was considered unable to tolerate a doublet regimen, chemotherapy with capecitabine plus bevacizumab was introduced as first-line chemotherapy, as is typical for frail patients who are unsuitable candidates for intensive chemotherapy, according to the Japanese guidelines for unresectable advanced CRC (10).

Several concerns have risen about chemotherapy for elderly CRC patients owing to age-related comorbidities and functional status (11). Targeted agents, such as bevacizumab have been widely used for the treatment of metastatic CRC combined with chemotherapy (12). Bevacizumab, which has several side-effects, needs careful consideration when used for the treatment of elderly patients. The PRODIGE 20 study which evaluated chemotherapy associated with bevacizumab in patients aged 75 years or older demonstrated that bevacizumab in combination with both 5-fluorouracil monotherapy or doublet chemotherapy is well-tolerated and efficient in selected elderly patients (13). Longer tumour control is observed with chemotherapy plus bevacizumab than with chemotherapy only. Furthermore, normal independent activity, a positive daily living score, and no previous cardiovascular disease may predict good efficacy and safety 


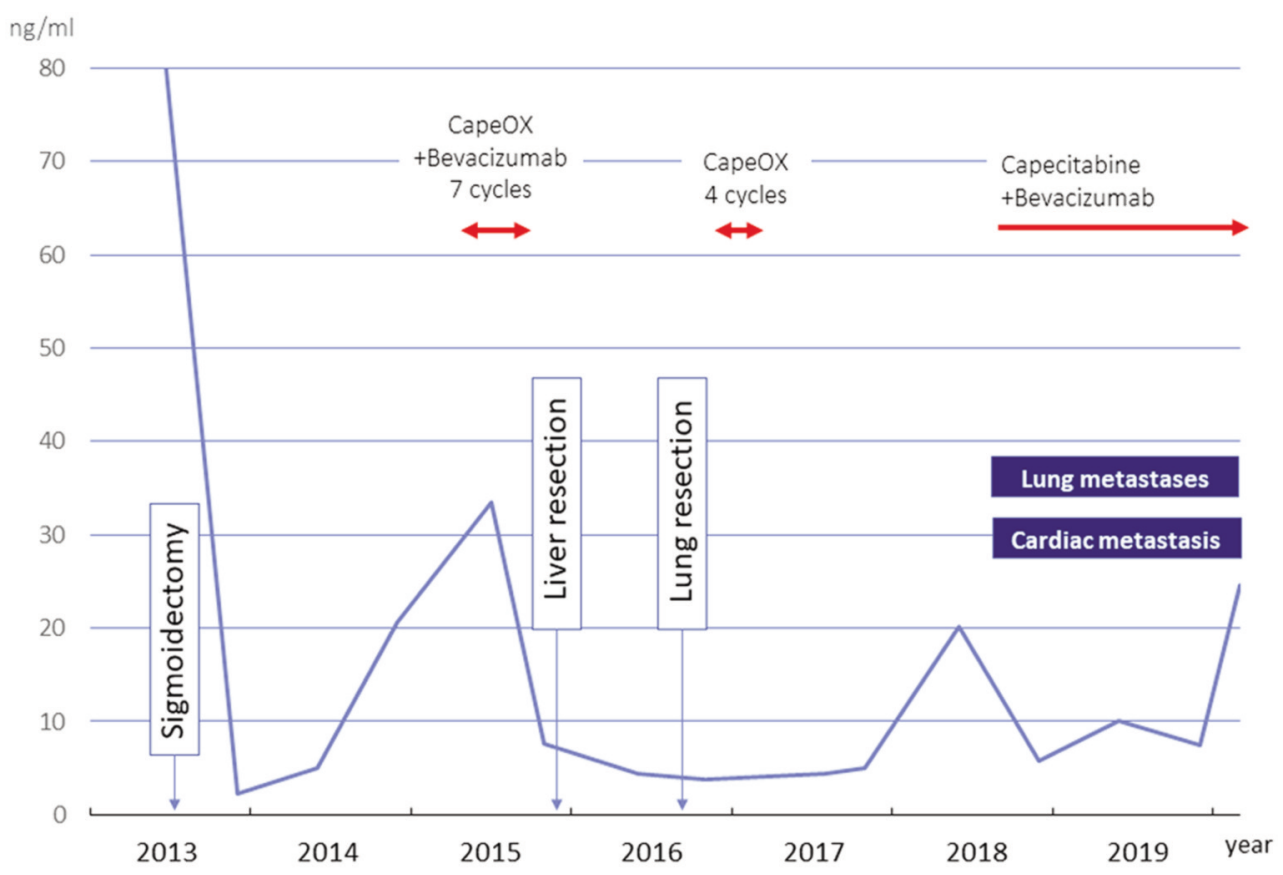

Figure 3. The clinical course of the patient and the carcinoembryonic antigen level.

of both bevacizumab and chemotherapy in elderly patients with metastatic CRC (14). Because this case met the above points, bevacizumab combination regimen is considered promising in this case. Additionally, a phase III trial comparing capecitabine alone or combined with bevacizumab for elderly patients (AVEX trial) demonstrated that the combination of bevacizumab and capecitabine is an effective and well-tolerated regimen for elderly patients with metastatic CRC (15). Progression-free survival was significantly longer in the capecitabine plus bevacizumab group than in the capecitabine alone group [median $=9.1$ months $(95 \% \mathrm{CI}=7.3$ 11.4) vs. 5.1 months (95\% $\mathrm{CI}=4.2-6.3) ; \mathrm{HR}=0.53(95 \%$ $\mathrm{CI}=0.41-0.69) ; p<0.0001]$. However, as a demerit to choose a capecitabine plus bevacizumab regimen as first-line chemotherapy, there is a possibility that the following effective drugs may not be used up. These drugs include irinotecan, which has never been administered before in the diagnosis of myocardial metastasis, and anti-EGFR drugs which may exert effect in RAS wild-type CRC. However, it should be noted that several studies have demonstrated that doublet chemotherapy has a limited effect on progression-free survival and does not prolong overall survival compared to fluoropyrimidine alone in elderly patients $(16,17)$.

The right atrium and ventricle of the heart remain the most common sites of metastasis in the heart in most cases suggestive of haematogenous spread (18). Previously, a higher frequency of right atrial metastases than of right ventricular metastases from CRC was reported (19). We speculate that micrometastatic lesions that enter the heart through the systemic blood first reach the atrium, where the atrial blood flow is slower than the ventricular blood flow. Therefore, cancer cells have the potential to easily engraft in the atrial endocardium and myocardium. We found only 12 cases of well-documented metastatic CRC that involved the right ventricle of the heart. Table I shows 13 reports including the present case $(3,6,9,19-21)$. The median age at presentation in these reports was 71 years (range $=35-77$ years) with a slight prevalence in female patients (seven patients, 53.8\%). Approximately half of the primary lesions were of the colic and rectal origins. Of the six cases in which tumour differentiation was described, there were four cases of moderately differentiated adenocarcinoma and one case of well differentiated adenocarcinoma and mucinous adenocarcinoma. Eight of the 13 cases were symptomatic, with symptoms such as dyspnoea and palpitation owing to congestive heart failure resulting from right ventricular outflow tract obstruction and valvular disorder. Solitary cardiac ventricular metastasis from CRC in the absence of lung and liver metastases is extremely rare (3). In this review, there were six cases with metastases other than cardiac metastasis at the time of the diagnosis of cardiac metastasis. Except for two cases whose treatment strategy is unknown, resection of cardiac metastasis and metastases other than cardiac metastasis was performed in one case. However, the outcome was death within 30 days after surgery, whereas chemotherapy was performed in three cases 


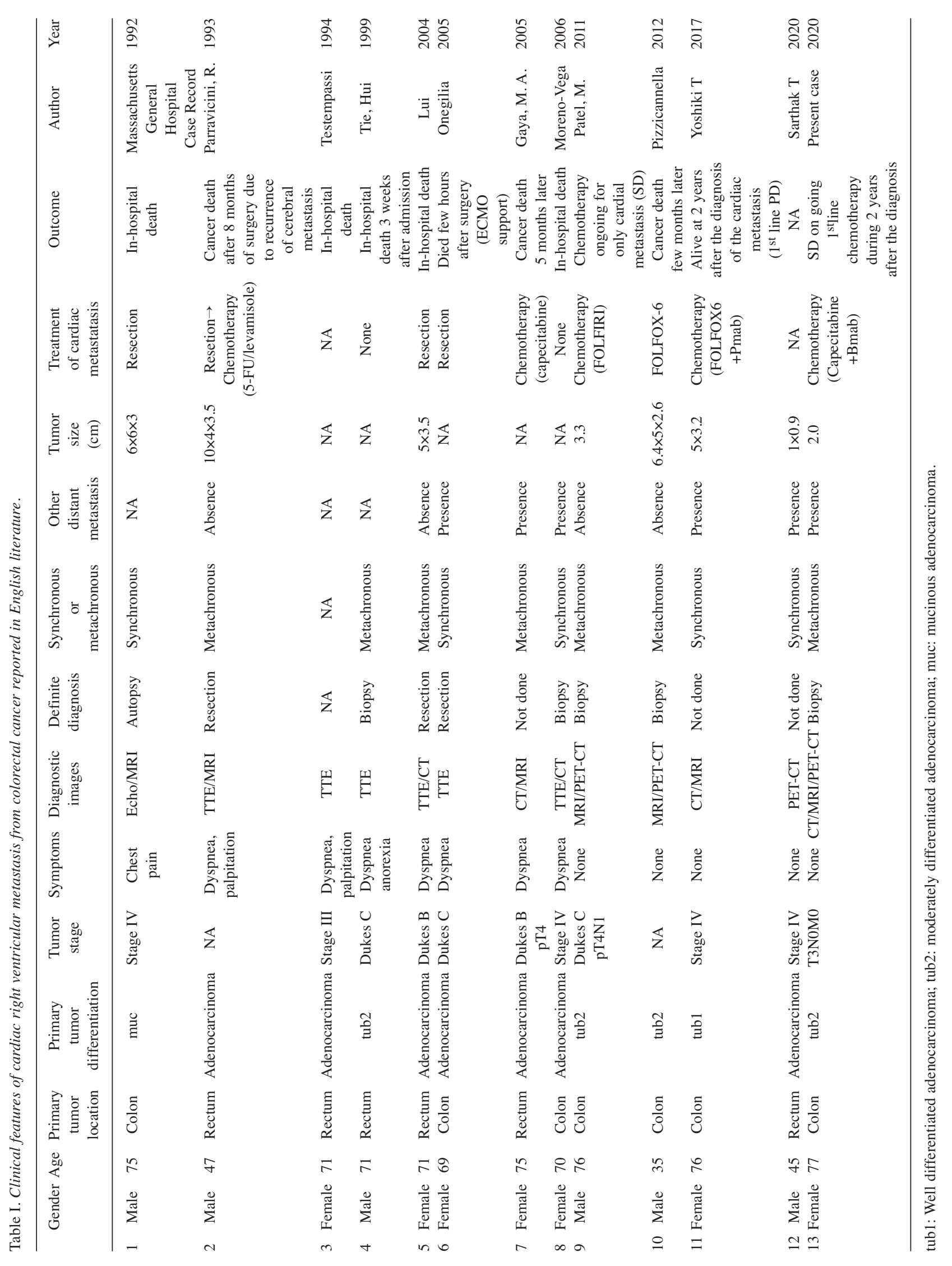


and a relatively good prognosis was achieved. However, resection was performed in two of four cases diagnosed only with cardiac ventricular metastasis. The outcome was death within 30 days after surgery in one case, whereas chemotherapy was effectively performed in the other two cases. Therefore, the surgical resection of the right ventricle may not be recommended owing to a high surgical risk and chemotherapy may be better than resection, especially in cases with metastases to other organs.

\section{Conclusion}

We encountered a patient who had cardiac right ventricular metastasis with lung metastases from CRC that could be controlled by capecitabine plus bevacizumab chemotherapy for a long time. The review of cardiac right ventricular metastasis from CRC demonstrated that the surgical risk of an operation to remove the right ventricle is high and not recommended. These findings suggest that chemotherapy may be suitable for selected patients with asymptomatic metastatic cardiac tumours; although to date, there are no standardised approaches to treatment in patients with cardiac metastases from CRCs. Further studies are needed to delineate the best treatment course in this group of patients.

\section{Conflicts of Interest}

The Authors declare that they have no conflicts of interest.

\section{Authors' Contributions}

SH, MM, SI: Drafted the manuscript; SH, MM, SI, HN, KS, TN, TI: contributed to patient care; SH, MM: performed the literature search; HE: advised about the oncological outcome; SH, MM, SI, TI: participated in the critical revision of the manuscript. All Authors read and approved the final manuscript.

\section{References}

1 Bussani R, De-Giorgio F, Abbate A and Silvestri F: Cardiac metastases. J Clin Pathol 60(1): 27-34, 2007. PMID: 17098886. DOI: $10.1136 /$ jcp.2005.035105

2 Sladden D, Yamagata K, Pllaha E and Busuttil W: Squamous cell carcinoma of unknown origin metastasising to the right atrium causing acute heart failure. BMJ Case Rep 2015, 2015. PMID: 26101300. DOI: 10.1136/bcr-2015-210042

3 Patel M, Neal CP, Berry DP and Garcea G: An isolated cardiac metastasis from colorectal adenocarcinoma. BMJ Case Rep 2011: bcr0820103270-b, 2011. PMID: 22715227. DOI: 10.1136/ bcr.08.2010.3270

4 Ayyala SS, Urcuyo DM, Kannarkatt PT, Kovacs JE and Terrigno $\mathrm{NJ}$ : A rare case of atrial metastasis from a rectal adenocarcinoma. J Clin Med Res 9(10): 886-888, 2017. PMID: 28912926. DOI: $10.14740 /$ jocmr $3123 \mathrm{w}$

5 Klatt EC and Heitz DR: Cardiac metastases. Cancer 65(6): 14561459, 1990. PMID: 2306690. DOI: 10.1002/1097-0142(1990 0315)65:6<1456::aid-cncr2820650634>3.0.co;2-5

6 Tripathy S, Parida GK, Naswa N, Jha P, Reddy S and Arun Raj ST: Right ventricle metastasis from carcinoma rectum: Findings on (18)f-fluorodeoxyglucose positron emission tomographycomputed tomography. Indian J Nucl Med 35(1): 78-79, 2020. PMID: 31949380. DOI: 10.4103/ijnm.IJNM_15_19

7 Koizumi J, Agematsu K, Ohkado A, Shiikawa A and Uchida T: Solitary cardiac metastasis of rectal adenocarcinoma. Jpn J Thorac Cardiovasc Surg 51(7): 330-332, 2003. PMID: 12892468. DOI: $10.1007 /$ bf02719389

8 Parravicini R, Fahim NA, Cocconcelli F, Barchetti M, Nafeh M, Benassi A, Grisendi A, Garuti W and Benimeo A: Cardiac metastasis of rectal adenocarcinoma. Surgical treatment. Tex Heart Inst J 20(4): 296-298, 1993. PMID: 8298329.

9 Tsujii Y, Hayashi Y, Maekawa A, Fujinaga T, Nagai K, Yoshii S, Sakatani A, Hiyama S, Shinzaki S, Iijima H and Takehara T: Cardiac metastasis from colon cancer effectively treated with 5fluorouracil, leucovorin, and oxaliplatin (modified folfox6) plus panitumumab: A case report. BMC Cancer 17(1): 152, 2017. PMID: 28228152. DOI: 10.1186/s12885-017-3147-2

10 Watanabe T, Muro K, Ajioka Y, Hashiguchi Y, Ito Y, Saito Y, Hamaguchi T, Ishida H, Ishiguro M, Ishihara S, Kanemitsu Y, Kawano H, Kinugasa Y, Kokudo N, Murofushi K, Nakajima T, Oka S, Sakai Y, Tsuji A, Uehara K, Ueno H, Yamazaki K, Yoshida M, Yoshino T, Boku N, Fujimori T, Itabashi M, Koinuma N, Morita T, Nishimura G, Sakata Y, Shimada Y, Takahashi K, Tanaka S, Tsuruta O, Yamaguchi T, Yamaguchi N, Tanaka T, Kotake K and Sugihara K: Japanese society for cancer of the colon and rectum (jsccr) guidelines 2016 for the treatment of colorectal cancer. Int J Clin Oncol 23(1): 1-34, 2018. PMID: 28349281. DOI: 10.1007/s10147-017-1101-6

11 Aparicio T, Pamoukdjian F, Quero L, Manfredi S, Wind P and Paillaud E: Colorectal cancer care in elderly patients: Unsolved issues. Dig Liver Dis 48(10): 1112-1118, 2016. PMID: 27260332. DOI: 10.1016/j.dld.2016.05.011

12 Mayer RJ: Targeted therapy for advanced colorectal cancermore is not always better. N Engl J Med 360(6): 623-625, 2009. PMID: 19196680. DOI: 10.1056/NEJMe0809343

13 Aparicio T, Bouche O, Taieb J, Maillard E, Kirscher S, Etienne PL, Faroux R, Khemissa Akouz F, El Hajbi F, Locher C, Rinaldi Y, Lecomte T, Lavau-Denes S, Baconnier M, Oden-Gangloff A, Genet D, Paillaud E, Retornaz F, Francois E, Bedenne L and for PI: Bevacizumab+chemotherapy versus chemotherapy alone in elderly patients with untreated metastatic colorectal cancer: A randomized phase ii trial-prodige 20 study results. Ann Oncol 29(1): 133-138, 2018. PMID: 29045659. DOI: 10.1093/ annonc/mdx 529

14 Aparicio T, Bouche O, Francois E, Retornaz F, Barbier E, Taieb J, Kirscher S, Etienne PL, Faroux R, Khemissa Akouz F, El Hajbi F, Locher C, Rinaldi Y, Lecomte T, Lavau-Denes S, Baconnier M, Oden-Gangloff A, Genet D, Bedenne L and Paillaud E, for PRODIGE 20 investigators: Geriatric analysis from prodige 20 randomized phase ii trial evaluating bevacizumab+chemotherapy versus chemotherapy alone in older patients with untreated metastatic colorectal cancer. Eur J Cancer 97: 16-24, 2018. PMID: 29777975. DOI: 10.1016/j.ejca.2018.03.030

15 Cunningham D, Lang I, Marcuello E, Lorusso V, Ocvirk J, Shin DB, Jonker D, Osborne S, Andre N, Waterkamp D and Saunders MP: Bevacizumab plus capecitabine versus capecitabine alone in elderly patients with previously untreated metastatic colorectal 
cancer (avex): An open-label, randomised phase 3 trial. Lancet Oncol 14(11): 1077-1085, 2013. DOI: 10.1016/s14702045(13)70154-2

16 Seymour MT, Thompson LC, Wasan HS, Middleton G, Brewster AE, Shepherd SF, O'Mahony MS, Maughan TS, Parmar M and Langley RE: Chemotherapy options in elderly and frail patients with metastatic colorectal cancer (mrc focus2): An open-label, randomised factorial trial. Lancet 377(9779): 1749-1759, 2011. DOI: 10.1016/s0140-6736(11)60399-1

17 Aparicio T, Lavau-Denes S, Phelip JM, Maillard E, Jouve JL, Gargot D, Gasmi M, Locher C, Adhoute X, Michel P, Khemissa F, Lecomte T, Provencal J, Breysacher G, Legoux JL, Lepere C, Charneau J, Cretin J, Chone L, Azzedine A, Bouche O, Sobhani I, Bedenne L, Mitry E and FFCD Investigators: Randomized phase iii trial in elderly patients comparing lv5fu2 with or without irinotecan for first-line treatment of metastatic colorectal cancer (ffcd 2001-02). Ann Oncol 27(1): 121-127, 2016. PMID: 26487578. DOI: 10.1093/annonc/mdv491

18 Choi PW, Kim CN, Chang SH, Chang WI, Kim CY and Choi HM: Cardiac metastasis from colorectal cancer: A case report. World J Gastroenterol 15(21): 2675, 2009. PMID: 19496202. DOI: $10.3748 /$ wjg. 15.2675
19 Bianchi G, Cerillo AG, Murzi M and Solinas M: Surgical resection of colorectal carcinomas metastatic to the heart. J Card Surg 31(11): 677-682, 2016. PMID: 27601061. DOI: 10.1111/ jocs. 12841

20 Tie H, Thorburn CW and Tran D: Cardiac metastasis from a rectal adenocarcinoma. Clin Cardiol 22(11): 749, 1999. PMID: 10554692. DOI: $10.1002 / \mathrm{clc} .4960221115$

21 Gaya MA, Randle A and Ashford RF: Right ventricular outflow tract obstruction secondary to myocardial metastases from colorectal cancer. Clin Oncol (R Coll Radiol) 17(1): 70-71, 2005. PMID: 15714938. DOI: 10.1016/j.clon.2004.11.001

Received June 22, 2020

Revised July 28, 2020

Accepted July 29, 2020 